\title{
FAST ROTATION CENTER IDENTIFICATION METHODS FOR VIDEO SEQUENCES
}

\author{
王嘉欣 $W O N G \mathrm{Ka}$ Yan \\ kywong@cs.hku.hk
}

\author{
葉志立 YIP Chi Lap \\ clyip@cs.hku.hk
}

\author{
Department of Computer Science, The University of Hong Kong, Pokfulam, Hong Kong
}

\begin{abstract}
Rotational motion can often be seen in video. However, comparatively little research has been done to investigate rotational motions in video, whose analysis could be useful. For example, if we can efficiently identify the rotation center of a spinning object, extraction and tracking of it can be made easier by grouping points moving at the same radial speed. It could also improve compression by synthesizing analyzed spin transitions, and help tracking of rotating objects. In this paper, we introduce a set of rotation center location methods using only the motion field constructed during video encoding, along with a few methods for improving their performances. These methods can be implemented using integer operations only. They are up to 1.81 times faster than the traditional circulation analysis method with little sacrifice in accuracy, and are not affected by asymmetric fields caused by translational motions.
\end{abstract}

\section{INTRODUCTION}

Motion compensated prediction is essential in blockbased video encoding standards such as H.261 and MPEG. A frame, or picture, is divided into blocks, and each block in the current picture is predicted from a spatially translated block in a reference picture. The amount of translation is specified by a motion vector. By encoding only the motion vector and the difference between the actual and predicted blocks, compression is achieved. As a side effect, a motion vector field is built for each picture. This vector field can be exploited for tasks like object extraction, object tracking, or scene change detection, where specialized and potentially computationally expensive algorithms are traditionally required. In this paper, we aim at developing fast algorithms that could simplify these tasks for scenes with rotating objects by using the motion vector field. Example applications of the algorithms include the extraction of rotating entities, tracking of rolling objects, and detection of scene changes by the spin effect.

Rotational motion can often be seen in video. The moving wheels of a car, skaters spinning on ice, the top view of a helicopter, or a high diver performing somersaults are but some examples of rotational motions that could be captured in video. Moreover, besides translational effects such as slide or push, video transitions also make use of rotation, as in the spin effect. However, comparatively little research has been done to investigate rotational motions in video, whose analysis could be useful. For example, if we can efficiently identify the rotation center of a spinning object, extraction and tracking of it can be made easier, as points of the object at the same distance from the center move at the same speed, and can be grouped together easily. The estimation of the spinning center can also assist in scene change detection. The detection of spin transition could allow videos to be compressed better by encoding only the scenes before and after the transition and regenerating the spin on the decoder side.

Existing rotating image analysis mainly focus on transformation techniques. In [1] and [2], Fourier transform is applied to estimate the rotation angle. Wavelet transform is also used to estimate the motion of rotating objects [3]. In [4], critical points of a motion field, such as swirl, vortices, sinks, or sources, are characterized and extracted. The idea is to represent the field as the sum of a solenoidal and an irrotational field, and assuming the field follows the Rankine model, the critical points are extracted by some mathematical analysis. Alternatively, critical points can be classified by examining the structure of the linear phase portrait matrix [5]. The analysis techniques introduced above are often computationally expensive, and often require a well structured motion field that fits the mathematical models to work well.

In Section 2, we introduce a set of novel motion field analysis methods for identifying rotation centers in video sequences. The traditional mathematical method of circulation analysis is also described. The new methods use only the motion vector field constructed during video encoding. By analyzing only the structure of the field, they are not affected by the asymmetry caused by combined translation and rotational motions. In Section 3, the experimental setup for evaluating the efficiency and effectiveness of these methods against circulation analysis is described. The results are presented in Section 4, followed by a summary in Section 5. 


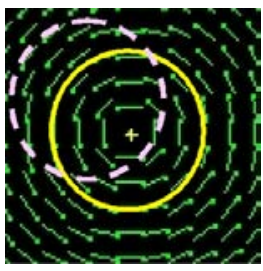

(a) Illustration of circulation analysis

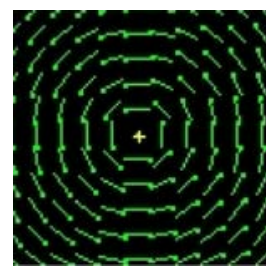

(b) Artificial circular motion field

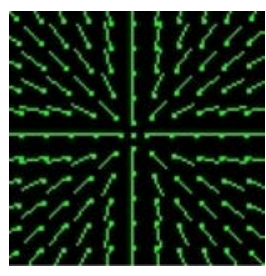

(c) Motion field of 1(b) rotated by $90^{\circ} \mathrm{CCW}$
Fig. 1. Illustration of circulation and straight line analysis

\section{ROTATION CENTER LOCATION}

In this section, we will describe the traditional mathematical method of circulation analysis and a set of novel methods, namely straight line analysis and sector analysis, for fast rotation center location by analyzing motion field structure. Conceptually, straight line analysis transforms the motion field and sector analysis takes care of the error caused by integer motion estimation algorithms. Both methods use integer operations only. Variations of giving weights to vectors and filtering them, which improve their efficiency and effectiveness, are also investigated.

\subsection{Circulation}

The tendency of fluids to circulate around a region is measured by circulation. It can be found by integrating along a closed curve $C$ the projection of motion vector onto the tangent of the curve, written $\oint_{C} \vec{u} \cdot \vec{T} d s$. Here, $\vec{u}$ is a motion vector in the vector field, and $\vec{T}$ is a unit tangent vector along $C$. For $C$ to be a circle of fixed radius, the rotation center can be located by placing it at a point that gives the maximum absolute circulation value. Figure 1(a) illustrates the idea of circulation analysis. The solid yellow curve which follows the motion field gives a higher circulation value than the pink dotted curve.

Circulation analysis uses search method to find the circle location that gives the maximum normalized circulation value. For a candidate center point, circles of different radii are used to calculate the circulation values. The values are normalized by the length of the circumference to avoid the domination caused by large circles. The maximum normalized circulation value among all radii is recorded and compared with that of other center point candidates. The point having the maximum circulation value is considered as the rotation center. The disadvantage of this method is that it involves floating point operations during integration.

\subsection{Straight line analysis}

Straight line analysis analyzes the structure of the field. We observe that by rotating each vector of a circular motion

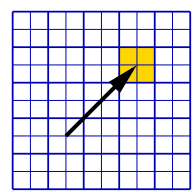

(a) Vector with \pm 0.5 pixel error

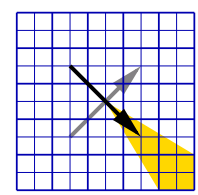

(b) Sector region from rotated vector

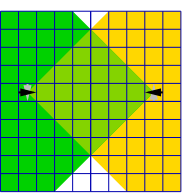

(c) Overlapping sectors from short vectors

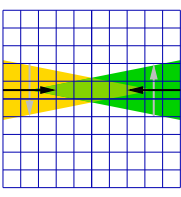

(d) Overlapping sectors from

long vectors
Fig. 2. Sector analysis method and short vector problem

field $90^{\circ}$ counterclockwise, a new field is formed with the vectors pointing towards the circulation center (Figures 1(b) and 1(c)). A set of straight lines formed by extensions of the rotated vectors can then be used to find the region the circulation center is in, as the highest number of straight lines would pass through it. Counting the number of straight lines passing through a region involves only integer operations, making it efficient. Combined translational and rotational motion would result in an asymmetric motion field. By considering only the directions of the vectors, the translation effect on the center region is minimized.

\subsection{Sector analysis}

Motion estimation algorithms usually give motion vectors with integer pixel coordinates in order to be fast. However, it would cause an error of \pm 0.5 pixel on each vector. That is, a vector can be seen to represent any vector within a range of \pm 0.5 pixel on each of its coordinate, one whose end point fall into the shaded region of Figure 2(a). Therefore, using the idea of straight line analysis, each straight line is turned into a sector as shown in Figure 2(b) (only the sector in the direction of vector is shown for simplicity). This sector region represents all possible straight lines formed by the set of vectors derived from a vector with integer coordinates. The circulation center is in an area covered by the highest number of sector regions.

\subsection{Sector analysis with weighting}

Vectors circulate around the rotation center. A vector which is far away from the rotation center may not belong to the rotating object. It is therefore not as significant as the ones that are close, and may also cause undesired effect or noise to the analysis. In order to minimize this effect, a weighting function is added to sector analysis. Two weighting functions are used for testing. The first uses the simple inverse weighting relationship, $w=\frac{1}{r}$, where $w$ is the weight and $r$ is the distance between the vector and the investigation point. The second uses a Gaussian formula, $w=\frac{1}{\sqrt{2 \pi} \sigma} e^{-\frac{r^{2}}{2 \sigma^{2}}}$, as a weighting function. In our experiment, $\sigma$ is chosen so that $80 \%$ of typical rotating object (diameter approximately $50 \%$ of screen width) is covered 
Table 1. Experimental video sequences

\begin{tabular}{l|r|l}
\hline Name & No. of frames & Characteristics \\
\hline \hline Toy & 105 & A toy with an object having pure rotation \\
\hline Spin & 45 & Video with spin transition effect \\
\hline Typhoon & 240 & $\begin{array}{l}\text { Sequence of radar images with a typhoon } \\
\text { having non-rigid rotational and translational motion }\end{array}$ \\
\hline
\end{tabular}

within one $\sigma$. In both cases, $w$ is set to 1 when $r=0$, as $r=0$ represents the point that is being investigated. These functions were designed so that a heavier weight is given to vectors close to the investigation point.

\subsection{Short vector filtering}

The \pm 0.5 pixel uncertainty is relatively large for short vectors. Short vectors would cause the lines to deviate from the center in straight line analysis, or the sectors to cover a large area in sector analysis (Figures 2(c) and 2(d)). To reduce this effect, variations of the methods in which vectors with a length shorter than shortLength pixels ( 3 in our experiments) are filtered out.

\section{EXPERIMENTAL DESIGN}

To evaluate the performance of our methods, a Javabased system prototype is built. Given a video, its frames are extracted for motion field construction. Videos with only one rotating object are used as our methods can be easily adapted to handle multiple objects. Video frames are segmented into a number of square blocks with length blockSize pixels spaced stepSize pixels apart on both dimensions. Motion compensated prediction is applied to derive motion vector of each block. The distortion function used is Mean Absolute Error, and Three Step Search is used as the motion vector searching algorithm. A $5 \times 5$ median filter is then applied to smooth out the motion field. The motion field is then analyzed using the methods described in Section 2 for the proposed rotation center.

Three video sequences with specific features, as shown in Table 1, were used for testing. The effectiveness of the center location methods are evaluated by comparing it with the actual rotation centers of the object in the videos. To evaluate the efficiency of the methods, a profiling test is done on a Macintosh PowerBook G4 with $1 \mathrm{GHz}$ PowerPC G4 processor and 512MB RAM running Mac OS X. In our experiments, the parameters blockSize and stepSize are set to 16 and 10 pixels respectively.

\section{RESULTS AND ANALYSIS}

To evaluate the performance of the center location algorithms for objects with pure rotation, the Toy video is used.

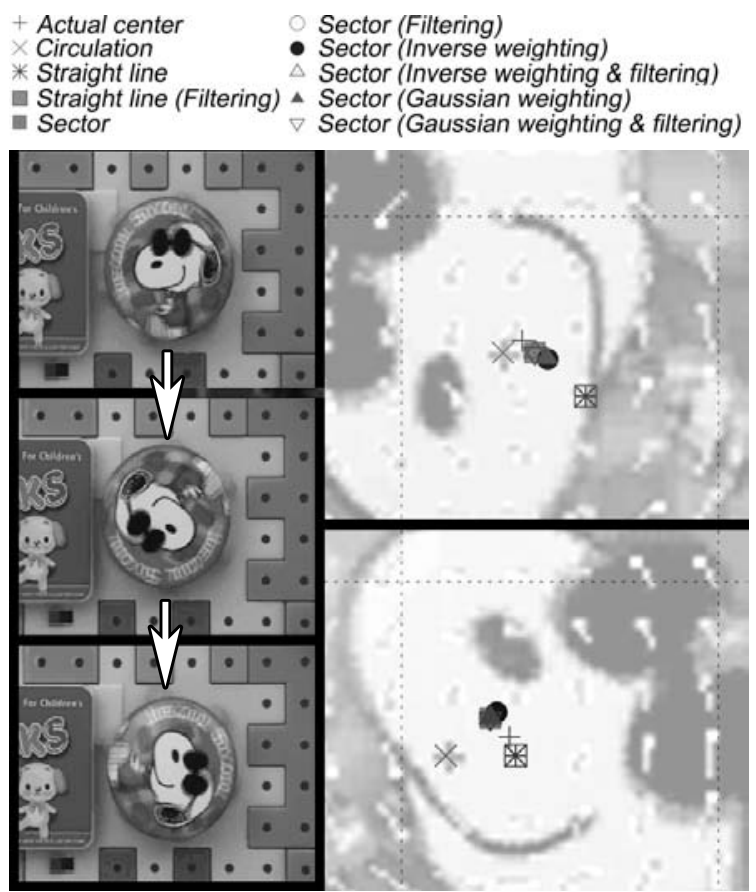

(a) Pure rotation (left: sample frames, right: results zoomed in)

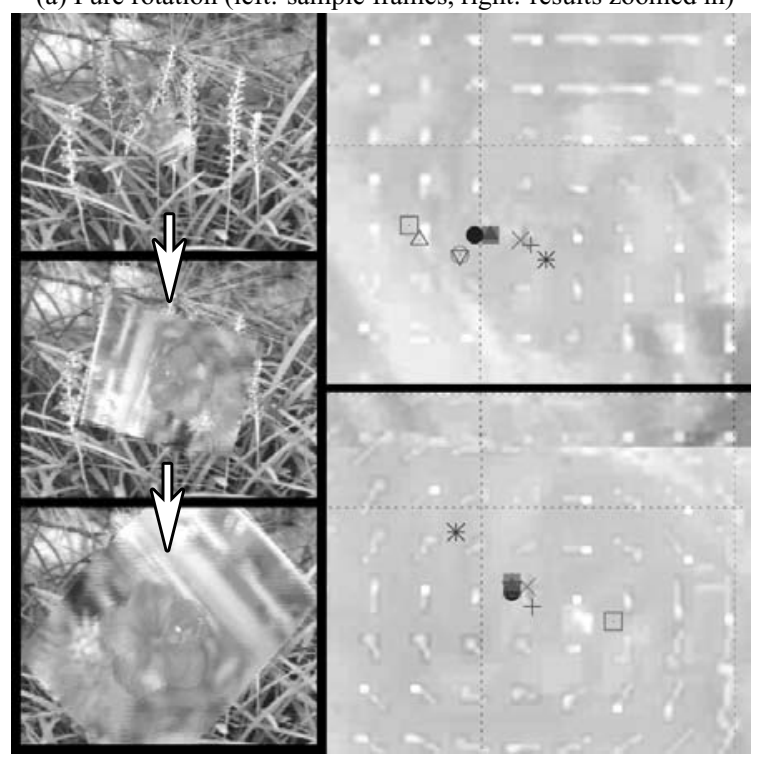

(b) Spin transition (left: sample frames, right: results zoomed in)

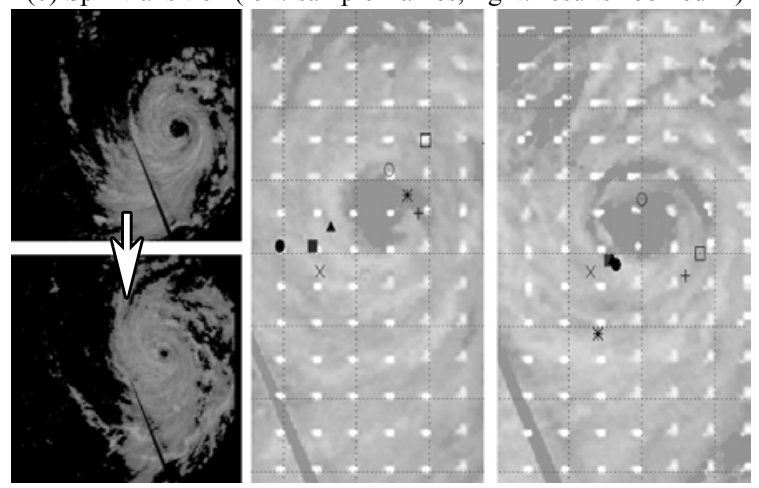

(c) Typhoon tracking (left: sample frames, right: results zoomed in)

Fig. 3. Experimental results 
Table 2. Percentage of centers located within a step size

\begin{tabular}{l|r|r}
\hline Method & Rotation & Scene change \\
\hline \hline Circulation & $99.0 \%$ & $100.0 \%$ \\
\hline Straight line & $81.0 \%$ & $73.3 \%$ \\
Sector & $95.2 \%$ & $88.9 \%$ \\
Sector with weighting (Inverse) & $93.3 \%$ & $84.4 \%$ \\
Sector with weighting (Gaussian) & $95.2 \%$ & $95.6 \%$ \\
\hline Straight line with filtering & $81.9 \%$ & $68.9 \%$ \\
Sector with filtering & $99.0 \%$ & $86.7 \%$ \\
Sector with weighting (Inverse) and filtering & $98.1 \%$ & $82.2 \%$ \\
Sector with weighting (Gaussian) and filtering & $98.1 \%$ & $91.1 \%$ \\
\hline
\end{tabular}

Figure 3(a) shows two of the frames with the proposed rotation centers found by different methods. Since the density of the motion field affects the accuracy of the rotation center location, we evaluate it by checking the percentage of frames having proposed centers within a step size in the whole video sequence. Table 2 shows the statistics. All the methods give proposed centers with a correct rate of more than $81 \%$. In particular, all but one variant of sector analysis methods give proposed centers within a step size in more than $95.2 \%$ of the frames. The statistics also show that motion field analysis methods with short vector filtering give a better correct rate. Sector methods with filtering all give more than $98.1 \%$ correctness. These experiments give a basic validation of the methods for videos with pure rotation.

The Spin video is used for evaluating the effectiveness of the methods in assisting scene change detection. Figure 3(b) shows sample results, and Table 2 shows the statistics. Similar to the Toy case, sector analysis performs better than straight line analysis. Short vector filtering, however, cannot enhance the results. In the spinning transition, the scene that replaces the original one spins while zooming out from the center. Early in the transition, the new scene is not large enough for motion compensation prediction algorithm to find vectors long enough to withstand the filtering. Though later in the transition the motion field built provides enough information for the analysis, the overall accuracy of the methods with filtering has already been worsened.

To illustrate that the methods can assist in object tracking, the Typhoon video is used. Figure 3(c) shows two frames of results. It is found that all the methods give satisfactory results, reporting center locations near the typhoon eye. Typhoons are nonrigid bodies undergoing rotational and translational motions, which make its tracking difficult. Practical typhoon eye location involves operations such as image preprocessing, region of interest estimation, and result postprocessing. The details can be found in [6].

Our profiling tests show that on average, straight line and sector analysis methods run 1.81 times and 1.61 times faster than circulation respectively. One of the reasons is that our methods can be implemented to use integer operations only, as opposed to floating point which are required by the circulation method. With nearly 100\% accuracy, straight line analysis and sector analysis are methods that give good tradeoff between efficiency and accuracy.

\section{SUMMARY AND FUTURE WORK}

A set of rotation center location methods using the motion field constructed during video encoding is proposed. The methods, namely straight line analysis and sector analysis, analyze the motion field structures. They are not affected by asymmetric fields caused by translational motions, and can be implemented using integer operations only. Vector weighting and short vector filter can be used to improve the performance. It was found that they are 1.61 to 1.81 times faster than the traditional circulation analysis method. All but one variant of sector analysis methods give proposed centers within a step size in more than $95.2 \%$ of the test video frames. These methods have potential applications in object extraction, tracking, and scene change detection, among others. Further improvements include fast detection of multiple rotating objects in video sequences, and the handling of different kinds of video transitions with rotation, such as twirl and whirlpool effects.

\section{ACKNOWLEDGMENT}

The authors thank the Hong Kong Observatory for their provision of tropical cyclone data for experiments.

\section{REFERENCES}

[1] S. Alliney, "Digital analysis of rotated images," IEEE Trans. PAMI, vol. 15, no. 3, pp. 499-504, 1993.

[2] Q. Wang and R. J. Clarke, "Estimation of general 2D motion using Fourier descriptors," in IEE Proc. on Vision, Image and Signal Processing, 1994, pp. 115-121.

[3] M. Kong, J.-P. Leduc, B. Ghosh J. Corbett, and V. Wickerhauser, "Wavelet based analysis of rotational motion in digital image sequences," in Proc. ICASSP, 1998, pp. 2777-2780.

[4] T. Corpetti, É. Mémin, and P. Pérez, "Extraction of singular points from dense motion fields: An analytic approach," Journal of Mathematical Imaging and Vision, vol. 19, pp. 175-198, 2003.

[5] C. F. Shu and R. C. Jain, "Vector field analysis for oriented patterns," IEEE Trans. PAMI, vol. 16, no. 9, pp. 946-950, Sept. 1994.

[6] Ka Yan Wong and Chi Lap Yip, "A motion field analysis systems for tropical cyclone eye fix," in International Conference on Systems, Man and Cybernetics, 2005, (to appear). 